\title{
RMCE-based insect cell platform to produce membrane proteins captured on HIV-1 Gag virus-like particles
}

\author{
João Vidigal ${ }^{1,2} \cdot$ Bárbara Fernandes $^{1,2} \cdot$ Mafalda M. Dias ${ }^{1,2}$ - Marco Patrone ${ }^{3}$. \\ António Roldão $^{1,2}$ • Manuel J. T. Carrondo ${ }^{1,2}$ • Paula M. Alves ${ }^{1,2}$ • Ana P. Teixeira ${ }^{1,2,4}$
}

Received: 22 August 2017 / Revised: 3 November 2017 / Accepted: 5 November 2017 / Published online: 16 November 2017

(C) Springer-Verlag GmbH Germany, part of Springer Nature 2017

\begin{abstract}
Conformationally complex membrane proteins (MPs) are therapeutic targets in many diseases, but drug discovery has been slowed down by the lack of efficient production tools. Co-expression of MPs with matrix proteins from enveloped viruses is a promising approach to obtain correctly folded proteins at the surface of virus-like particles (VLPs), preserving their native lipidic environment. Here, we implemented a site-specific recombinase-mediated cassette exchange (RMCE) strategy to establish a reusable HIV-1 Gagexpressing insect cell line for fast production of target MPs on the surface of Gag-VLPs. The Sf9 cell line was initially tagged with a Gag-GFP-expressing cassette incorporating two flipase recognition target sites (FRTs), one within the fusion linker of Gag-GFP. The GFP cassette was afterwards replaced by a Cherry cassette via flipase (Flp) recombination. The fusion of Gag to fluorescent proteins enabled high-throughput screening of cells with higher Gag expression and Flpmediated cassette exchange ability, while keeping the functionality of the VLP scaffold unaltered. The best cell clone
\end{abstract}

Electronic supplementary material The online version of this article (https://doi.org/10.1007/s00253-017-8628-3) contains supplementary material, which is available to authorized users.

Ana P. Teixeira

anat@itqb.unl.pt

1 IBET, Instituto de Biologia Experimental e Tecnológica, 2780-901 Oeiras, Portugal

2 Instituto de Tecnologia Química e Biológica António Xavier, Universidade Nova de Lisboa, 2780-901 Oeiras, Portugal

3 Biocrystallography Unit, San Raffaele Scientific Institute, 20142 Milan, Italy

4 Department of Biosystems Science and Engineering, ETH Zurich, Mattenstrasse 26, 4058 Basel, Switzerland was then Flp-recombinated to produce Gag-VLPs decorated with a human $\beta 2$-adrenergic receptor ( $\beta 2 \mathrm{AR})$. Release of a fluorescently labeled $\beta 2 \mathrm{AR}$ into the culture supernatant was confirmed by immunoblotting, and its co-localization with Gag-VLPs was visualized by confocal microscopy. Furthermore, the differential avidity of $\beta 2 A R$-dsplaying Gag-VLPs versus "naked" Gag-VLPs to an anti- $\beta 2 A R$ antibody measured by ELISA corroborated the presence of $\beta 2 A R$ at the surface of the Gag-VLPs. In conclusion, this novel insect cell line represents a valuable platform for fast production of MPs in their native conformation, which can accelerate small-molecule and antibody drug discovery programs.

Keywords Membrane proteins $\cdot$ GPCRs $\cdot$ Virus-like particles (VLPs) $\cdot$ RMCE systems $\cdot$ Insect cell line development

\section{Introduction}

Complex transmembrane proteins such as G-protein-coupled receptors (GPCRs) have been implicated in a multitude of diseases, including cancer and immune and inflammatory diseases, constituting an important target class for smallmolecule or antibody drug development (Huang et al. 2016; Hutchings et al. 2017). However, these proteins are often found at very little amounts in native tissues, and not only their heterologous overexpression is very challenging, but also they rely heavily on a lipidic environment to preserve their conformation, making them hardly to purify at relevant quantity and quality to succeed in drug discovery (Zhao and $\mathrm{Wu}$ 2012). Immunization strategies based on synthetic peptides, protein ectodomains, purified membrane proteins, membrane preparations, or whole cells have had limited success in generating conformational antibodies against many membrane proteins (Hutchings et al. 2010; Takeda et al. 2015). New 
technologies providing high concentrations of complex multispanning membrane proteins, with their conformational structure intact, are therefore needed.

Non-infectious virus-like particles (VLPs) have been proposed as efficient antigen-presenting platforms, capitalizing on the budding capacity of enveloped viruses. In particular, the main structural protein of the Retroviridae family, the Gag protein, has been extensively used as a VLP scaffold to display a variety of membrane proteins, either to be used as vaccines or in in vitro assays, including screening of binding molecules (Chua et al. 2013; Hoffman et al. 2000; Kirchmeier et al. 2014; Patrone et al. 2016; Pushko et al. 2017). The Gag protein self-assembles into VLPs and buds from the host cell in plasma membrane lipid rafts without the need of any additional viral factor (Delchambre et al. 1989). Among the chimeric GagVLPs which have been proposed as vaccines are particles with viral antigens from influenza, dengue, West Nile virus, human cytomegalovirus, and Rift Valley fever virus (Chua et al. 2013; Kirchmeier et al. 2014; Pushko et al. 2017). These pseudotyped VLPs are safer than live-attenuated vaccines and elicit robust and broad immune responses as target epitopes are displayed on their surface in a clustered fashion, efficiently activating antigen-presenting cells (Haynes et al. 2009; Ulmer et al. 2006). Owing to this feature, recombinant cancer antigens have been also displayed in Gag-VLPs to be used in cancer immunotherapy (Kurg et al. 2016). Furthermore, the use of VLPs as antigen vehicles for in vitro isolation of functional binding molecules from compound libraries can be particularly useful when looking for inhibitors for highly pathogenic viruses (e.g., H5N1 influenza virus), avoiding the use of live viruses for obvious biosafety reasons.

The insect cell-baculovirus expression system (IC-BEVS) has been widely explored for production of pseudotyped GagVLPs (Cruz et al. 2000; Haynes et al. 2009; Pushko et al. 2017), mainly because it provides eukaryotic post-translational modifications together with short timeframes from cloning the genes of interest until protein production (Fernandes et al. 2013). However, the IC-BEVS encloses a number of limitations in what concerns the production of complex membrane proteins. For example, the lytic nature of the infection process commonly leads to the release of host and viral proteases into the medium as culture progresses, impacting negatively on product quality. The concurrent production of baculovirus proteins can hamper the secretory pathway, reducing product quantity and quality. In addition, repeated rounds of baculovirus amplification within the host cell during production phase tend to generate defective interfering particles with concomitant reduction in protein yields (van Oers 2011).

Stable insect cell lines are an attractive alternative to the ICBEVS for the production of secreted and membrane-anchored proteins (Harrison and Jarvis 2016). To circumvent the laborious traditional cell line development process, our group has established a flipase (Flp)-mediated cassette exchange
(RMCE) system in an insect Sf9 cell line for targeted gene integration (Fernandes et al. 2012). By having a tagging cassette anchored in a given chromosomal locus, one could exchange it by a target cassette encoding the gene of interest flanked by the same pair of Flp recognition target (FRT) sites via Flp recombination. This allows for repeated use of the same locus for production of different proteins thus circumventing extensive clone screening (Qiao et al. 2009; Turan et al. 2013; Turan et al. 2014). In this work, we propose a novel RMCE strategy for fast development of an insect S9 cell line dedicated to produce enveloped VLPs pseudotyped with membrane proteins of interest. The design of efficient tagging and target cassettes and the combination of a twostep tagging process with fluorescence-activated cell sorting (FACS) allowed high-throughput screening of loci supporting high HIV-1 Gag expression and Flp recombination. As proof of concept, Gag-VLPs displaying a model membrane protein, the G-protein coupled $\beta 2$-adrenergic receptor ( $\beta 2 \mathrm{AR}$ ), were produced in this platform.

\section{Materials and methods}

\section{Plasmid construction}

The RMCE plasmids were constructed using the pTag 2.0 backbone (GenScript; GenBank MG356850), composed by the following elements: the OpIE2 promoter from the Orgyia pseudotsugata multicapsid nucleopolyhedrosis virus (OpMNPV) immediate-early 2 (ie-2) gene, FRT wt (Fwt) sequence, enhanced green fluorescent protein $(e g f p)$ gene with a polyA from the OpMNPV ie-2 gene, hygromycin gene, FRT F5 (F5) sequence, and the OpIE1 promoter. The polymerase chain reactions (PCRs) were carried out using the Phusion DNA Polymerase (Thermo Fisher Scientific), and cloning reactions were conducted using the In-Fusion HD Cloning Plus kit (Clontech). All inserts were verified by Sanger sequencing.

To assemble the tagging cassette (GenBank MG356851), we designed a new HIV-1 Gag fusion gene and inserted it in between the OpIE2 promoter and the $e G F P$ gene of the pTag 2.0. The HIV-1 Gag gene was cloned from pOET-Gag (Patrone et al. 2016), but was truncated in the stop codon (TAA), and followed by a FRT linker region composed by three GGGGS sequences on both sides of the Fwt site.

The target cassette encoding a red reporter protein (pTarget-Cherry; GenBank MG356852) was assembled from the pTagging. First, the gag gene and the OpIE1 and OpIE2 promoters were removed by inverted PCR. Then, the sequence from Fwt to F5 was cloned again into the backbone using the In-Fusion HD kit. The eGFP and hygromycin genes were removed by inverted PCR and replaced by $i$ Cherry and neomycin genes, respectively. Finally, the hr5AcIE1 promoter (cloned from pIEX-10, Merck) and the 
iFLP gene (from the OpIE2-iFlp-IEterm plasmid (GenBank MG051710), containing a Spodoptera frugiperda codonoptimized Flp gene kindly provided by the Protein Sample Production Facility (PSPF) at the Helmholtz Centre for Infection Research (Braunschweig, Germany), were cloned between the iCherry and neomycin genes.

The target cassette encoding a membrane protein (pTarget- $\beta 2$ AR; GenBank MG356853) was assembled from pTarget-Cherry. The fusion gene $\beta 2 A R$ was cloned from pCDNA_B2AR-i-pep, kindly provided by Sivaraj Sivaramakrishnan (Addgene plasmid \#47436). The iCherry gene was substituted by a codon stop with inverted PCR and cloned using the In-Fusion HD kit. Then, the neomycin gene was replaced by zeocin from pIZT/V5-His (Thermo Fisher Scientific) using PCR and the In-Fusion HD kit.

\section{Cell line and culture media}

Insect Sf9 cells (Invitrogen) were maintained in Sf-900II SFM medium (Gibco Invitrogen Corporation) in 125 - or $500-\mathrm{mL}$ shake flasks ( $10 \%$ working volume $-w / v)$, and routinely subcultured to $0.5 \times 10^{6}$ cells $/ \mathrm{mL}$ every $3-4$ days when the cell density reached $3-4 \times 10^{6}$ cells $/ \mathrm{mL}$. The cultures were agitated at $100 \mathrm{rpm}$ in an Innova 44R incubator (Eppendorf) at $27^{\circ} \mathrm{C}$. The cell density and viability were analyzed daily by the trypan blue exclusion method, in a Fuchs-Rosenthal hemocytometer chamber.

\section{Cell transfection with the tagging cassette}

To randomly integrate the tagging cassette in the genome of Sf9 cells, we transfected them with $0.3 \mu \mathrm{g}$ of pTagging, using $8 \mu \mathrm{L}$ of Cellfectin® II Reagent (Invitrogen) per $1 \times 10^{6}$ cells. Antibiotic selection started $72 \mathrm{~h}$ post transfection (hpt) in medium containing Hygromycin B (200 U/mL; InvivoGen).

\section{Recombinase-mediated cassette exchange}

Flp-mediated cassette exchange was performed in shake flask cultures, using a protocol described elsewhere (Fernandes et al. 2012). The medium was replaced $24 \mathrm{hpt}$, and the antibiotic selection marker (G418 $300 \mathrm{U} / \mathrm{mL}$ or Zeocin $200 \mathrm{U} / \mathrm{mL}$ (InvivoGen)) was added $72 \mathrm{hpt}$. When the cell viability dropped below 50\% (about $120 \mathrm{hpt}$ ), the cells were transferred to six-well plates and kept in selection.

\section{Production of Gag-VLPs}

The production of Gag-GFP, Gag-iCherry, and Gag- $\beta 2 \mathrm{AR}$ VLPs was performed in 500-mL shake flasks $(10 \% \mathrm{w} / \mathrm{v})$. Briefly, the engineered Sf9 cells were seeded at $0.5 \times 10^{6}$ cells/mL and kept in culture (as described above) until cell viabilities dropped below $80 \%$. The cell culture bulks were clarified, and the Gag-VLPs were concentrated using a twostep ultracentrifugation procedure: $40,000 \times g, 4{ }^{\circ} \mathrm{C}$ for $3 \mathrm{~h}$ followed by $60,000 \times g, 4{ }^{\circ} \mathrm{C}$ for $3 \mathrm{~h}$ on a layer of $20 \%(v / v)$ sucrose in PBS. The pellet containing the VLPs was collected and stored at $4{ }^{\circ} \mathrm{C}$ until further analysis.

\section{Analytics}

\section{Fluorescence microscopy}

Engineered Sf9 cells expressing the fusion proteins Gag-GFP or Gag-iCherry, or co-expressing Gag-iCherry and $\beta 2 \mathrm{AR}$-citrine, were visually inspected by fluorescence microscopy (DMI6000, Leica) and point scan confocal microscopy (SP5, Leica). During confocal image acquisition, each fluorophore was detected separately to avoid channel bleedthrough. The ImageJ software was used to merge channels and adjust the linear brightness and contrast of the images.

\section{Western blot}

Proteins were denatured at $70{ }^{\circ} \mathrm{C}$ for $10 \mathrm{~min}$, separated under reducing conditions in a $4-12 \%$ SDS gel (Thermo Fisher Scientific), and transferred to a nitrocellulose membrane using iBlot ${ }^{\circledR}$ Transfer Stack (Thermo Fisher Scientific). For Gag identification, we used the anti-HIV-1 p24 mouse monoclonal antibody (Abcam, Cat. ab9071) at a dilution of 1:2000. For identification of $\beta 2 \mathrm{AR}$, anti-HA (Abcam, Cat. ab18181) at a dilution of 1:1000 was used. As secondary antibody, we used the anti-mouse IgG antibody conjugated with horseradish peroxidase labeling (HRP) at a dilution of 1:5000 (Sigma, Cat. A9917). Protein band detection was performed with the enhanced chemiluminescence detection system (ECL) (Amersham Biosciences).

\section{Transmission electron microscopy}

Negative staining TEM was used to assess the conformation and size of Gag-GFP and Gag- $\beta 2$ AR VLPs. Briefly, $10 \mu \mathrm{L}$ of VLP samples (concentrated by ultracentrifugation) were fixed for $1 \mathrm{~min}$ in a copper grid coated with Formvar carbon (Electron Microscopy Sciences). Grids were washed with Milli-Q water and then stained with $1 \%(v / v)$ uranyl acetate for $2 \mathrm{~min}$ and left to air dry. Samples were then observed in a Hitachi H-7650 Transmission Electron Microscope (JEOL).

\section{Enzyme-linked Immunosorbent assay}

The concentration of Gag-VLPs was assessed using the LentiX p24 Rapid Titer Kit (Clontech) according to the manufacturer's instruction. To confirm that $\beta 2 \mathrm{AR}$ proteins were being displayed on the surface of Gag-VLPs, a modified/nonquantitative ELISA was performed. Briefly, 96-well 
MaxiSorp plates (Nunc) were coated with $5 \times 10^{9}$ purified Gag-VLPs (naked or coated with $\beta 2 \mathrm{AR}$ ) and incubated overnight at $4{ }^{\circ} \mathrm{C}$. After extensive plate washing and blocking with $4 \%(w / v)$ skimmed milk (Merck Millipore), we added an antiHA antibody (Abcam, Cat. ab18181) at 1:500 dilution and incubated for $2 \mathrm{~h}$ at room temperature. Then the plate was washed again and incubated for $2 \mathrm{~h}$ with an anti-mouse HRP conjugated antibody (1:5000 dilution). The reaction was visualized by the addition of the substrate TMB (Sigma) and stopped about $30 \mathrm{~min}$ later by adding $100 \mu \mathrm{L}$ of Stop Solution for TMB Substrates (Sigma). The absorbance of the wells was measured at $450 \mathrm{~nm}$ in a plate reader (Tecan).

\section{Fluorescence-activated cell sorting}

The populations of Sf9-GagGFP, Sf9-GagCherry, and Sf9Gag- $\beta 2$ AR cells were sorted in a MoFlo High-Speed Cell Sorter (Beckman Coulter) using a previously developed protocol (Vidigal et al. 2013). Single cells of the Sf9-GagCherry population were collected into 96-well plates (BD Falcon ${ }^{\mathrm{TM}}$ ) containing conditioned culture medium supplemented with 10\% FBS (Gibco) and Antibiotic-Antimycotic (Invitrogen), and monitored every 2-3 days. Wells containing multiple colonies were discarded. After adaptation to these culture conditions, robust colonies were obtained and expanded in Sf-900II SFM medium.

\section{Flow cytometry}

CyFlow R Space (Partec GmbH) was used to evaluate transfection and recombination efficiencies, and the fluorescence distributions of engineered Sf9 cells expressing Gag-GFP, Gag-iCherry, and Gag- $\beta 2$ AR-citrine. Exponentially growing cells were harvested and diluted in PBS (Gibco) to around $1 \times$ $10^{6} \mathrm{cell} / \mathrm{mL}$. Fluorescence intensity was collected from 10,000 cells per sample (at $509 \mathrm{~nm}$ for GFP and citrine, and at $610 \mathrm{~nm}$ for iCherry) and analyzed using the FlowJo Software (2009, Quantum Analysis GmbH).

\section{Real-time quantitative PCR}

The number of tagging cassettes integrated in the genome of the isolated cell clones was assessed using real-time quantitative PCR. Briefly, genomic DNA of $5 \times 10^{6}$ cells was isolated using the QIAamp® DNA Mini and Blood Mini Kit (Qiagen), according to the manufacturer's instructions, and quantified using a NanoDrop 2000C spectrophotometer (Thermo Scientific). As standard for quantitative PCR, we used the genome of a previously established Sf9 cell line from which we confirmed single copy integration of an OpIE2-containing cassette by Southern blot (Fernandes et al. 2012). Forward and reverse primer sequences for the OpIE2 promoter region (CATGATGATAAACAATGTATGGTGC and
GGGGAGGCCACCGAGTATAA) and 18S (AGGGTGTT GGACGCAGATAC and CTTCTGCCTGTTGAGGAACC) were designed. A master mix solution was prepared according to the LightCycler 480 SYBR Green I Master PCR kit (Roche Applied Science), and $18 \mu \mathrm{L}$ was distributed in each well of a 96-microwell plate, together with $2 \mu \mathrm{L}$ of DNA (equivalent to $200 \mathrm{ng}$ isolated DNA) or standard plasmid as PCR templates. The reactions were conducted in a LightCycler ${ }^{\circledR} 480$ Instrument (Roche Applied Science), and the qPCR results were analyzed using the LC480 software. Upon normalization to a housekeeping gene (18S), the number of integrated tagging copies per cell was estimated using the standard curve. Single-copy integration clones were considered to be those with normalized values between 0.6 and 1.45 .

\section{Results}

\section{Cell line development strategy}

In order to establish an $\mathrm{Sf} 9$ cell line to produce enveloped VLPs pseudotyped with membrane proteins of interest, we leveraged the capability of the HIV-1 Gag protein to bud through the plasma membrane and drag along anchored cell surface proteins. The cell line development strategy is based on Flp/FRT site-specific recombination and consists of five steps planned to facilitate the screening of loci supporting high expression and high recombination efficiency (Fig. 1a), with the aid of reporter genes and fluorescence-activated cell sorting (FACS). The tagging cassette expresses a fusion protein between Gag and eGFP, with their linker (Fig. S1) containing the upstream FRT site-Fwt (Fig. 1b). This will facilitate the screening of genomic "hot-spots" supporting high expression of Gag (step 2, Fig. 1b), while adding the possibility to remove the fused reporter gene whenever desired, by means of Flp-RMCE. The Fwt site is flanked by glycine-rich regions consisting of three repetitions of Gly-Gly-Gly-GlySer domains (Fig. S1). It was designed to increase flexibility and create spatial separation between Gag and the reporter protein, such that the VLP assembly is not impaired. In addition to the reporter gene, the region flanked by the FRT sites also harbors the resistance gene, and both will be replaced by different reporter and resistance markers at each recombination step of the cell line development process. The first recombination step is promoted in a FACS-enriched, high Gag-GFPexpressing cell population and aims at collecting those cells which are tagged in loci amenable to recombination (step 3, Fig. 1a). Placing the promoters driving the expression of the Gag-reporter protein fusion (OpIE2) and resistance markers (OpIE1) outside of the FRT recombination region (Fig. 1b) facilitates the isolation of cells which undergo successful RMCE, since it allows for both antibiotic selection and fluorescence sorting (step 4, Fig. 1a). This also ensures that most 

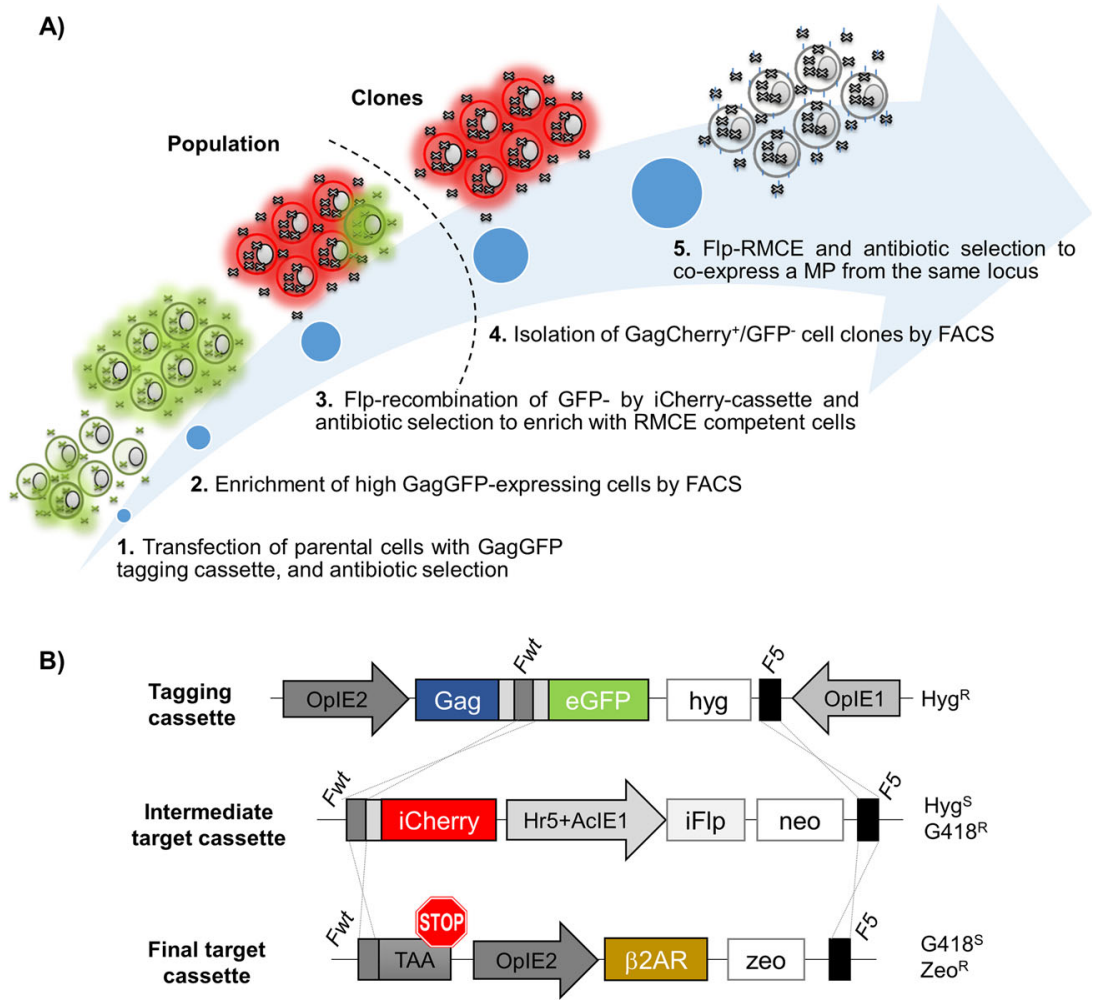

Fig. 1 Cell line development strategy based on Flp/FRT site-specific integration technology for co-expression of HIV-1 Gag and a target membrane protein from the same locus. a Five-step sequence from tagging parental Sf9 cells with a Gag reporter cassette (step 1), collecting high GFP-expressing cells by FACS (step 2), submitting cells to Flp-RMCE by a iCherry-encoding cassette to isolate cells tagged in loci amenable to recombination (step 3), until isolating high Gagproducer cell clones (step 4), which will co-express a membrane protein of choice from a single locus, in a process mediated by Flp (step 5). b Design of the cassettes. The tagging cassette contains an FRT wild-type (Fwt) and FRT 5 (F5), in which the Fwt site is part of a fusion linker between Gag and a GFP reporter protein (GAG-eGFP). The Gag gene and the OpIE2 and OpIE1 promoters are placed outside of the Flp recombination region. An intermediate cassette with the same pair of FRT sites flanking an Sf9 codon-optimized red reporter protein (iCherry), an Sf9 codon-optimized Flp (iFlp), and an hygromycin resistance gene, was designed to isolate by hyg selection and FACS a population of iCherry-expressing cells which are amenable to Flp recombination. The final target cassette has a stop codon downstream to the Fwt to eliminate the fluorescent protein from the Gag-VLPs when the producer cell line has been established, and encodes a human $\beta 2 \mathrm{AR}$ as model membrane protein and zeocin as resistance marker random genome integration events of the target cassettes are excluded during the selection process. We will validate the Sf9 cell platform by re-targeting a selected locus to produce GagVLPs displaying a model GPCR, the human $\beta 2$-adrenergic receptor ( $\beta 2 \mathrm{AR}$ ) (step 5, Fig. 1a).

\section{Establishment of a RMCE Gag-producing Sf9 cell population}

\section{Population tagged in high-expressing loci}

Parental Sf9 cells were transfected with the tagging cassette (Fig. 1b), and upon 3 weeks under hygromycin (Hyg) selection, a robust Sf9 cell population expressing Gag-GFP was obtained. Fluorescence microscopy confirmed the localization of Gag at the host cell membrane (Fig. 2a). Western blot analysis of Gag in the cell culture supernatant shows two bands: one at $83 \mathrm{kDa}$ representing the fusion of the Gag polyprotein and GFP, and a second at
$40 \mathrm{kDa}$ representing the cleavage products of the Gag polyprotein, the matrix (MA) and capsid (CA) proteins (Fig. 2b). Noteworthily, Gag seems to accumulate in the culture supernatant over time, reaching a peak level at $192 \mathrm{~h}$ of culture. To confirm the formation of correctly assembled Gag-GFP VLPs, cell culture supernatant samples were concentrated and analyzed by TEM. Spherical particles of external diameters of 100-120 nm could be observed (Fig. 2c), closely resembling the morphology (shape and size) of authentic HIV virions (Briggs et al. 2006). These results demonstrate that (i) Sf9 cell proteases can induce maturation of the Gag polyprotein, and more importantly (ii) the fusion of Gag to GFP with an FRT site composing the linker did not impair Gag processing into functional domains and its secretion as VLPs.

The stability of the Sf9 cell population expressing GagGFP was assessed by analyzing cell growth kinetics (Fig. 2d) and GFP fluorescence intensity (Fig. 2e) over several passages. During the first passages under Hyg 
A)

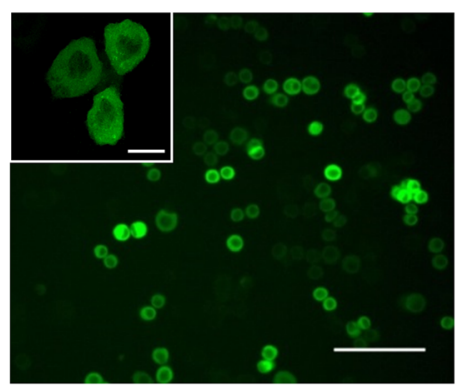

D)

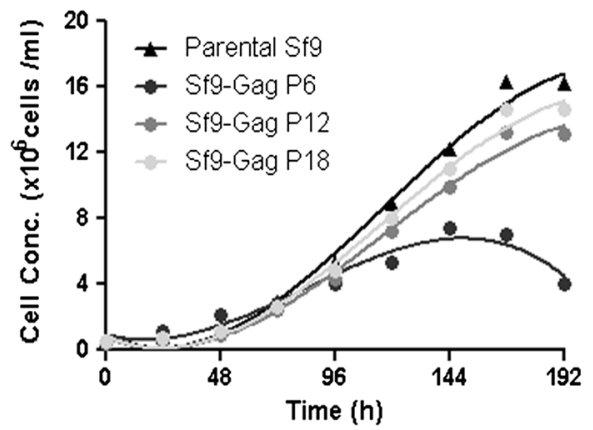

B)

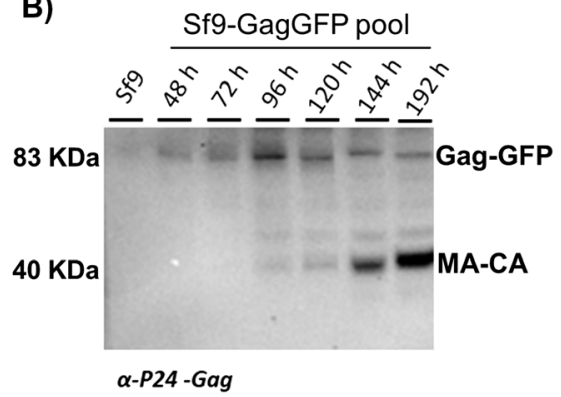

C)

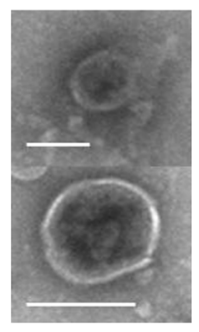

E)

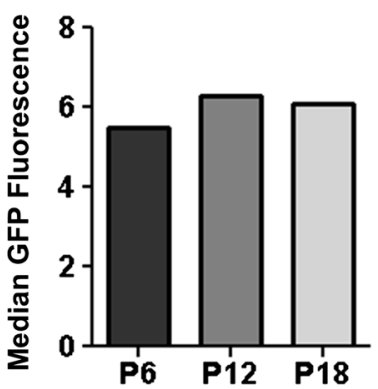

protein fused to GFP at $83 \mathrm{kDa}$ and its mature form (matrix (MA) and CA domains) at $40 \mathrm{kDa}$. $\mathrm{c}$ Electron microscopy images of the GagGFP VLPs pelleted by ultracentrifugation from the supernatant of GagGFPexpressing cells. Scale bar represents $100 \mathrm{~nm}$. d Growth profiles of parental Sf9 cells and engineered Sf9-GagGFP cells at different passages (P6, P12, and P18). e Median fluorescence intensity along passages analyzed by flow cytometry

Flp-RMCE in the tagging Sf9-GagGFP cell population

selection, cellular growth was impaired, but this effect ameliorated with successive cell passages and cells could reach similar growth kinetics of parental Sf9 cells. The median GFP fluorescence intensity of the cell population remained at similar levels independently of the passage number (Fig. 2e). Together, these results confirm the stable integration of the transgene over many cell generations, ruling out any potential cytotoxic effect arising from the continuous expression of Gag-GFP VLPs in Sf9 cells.

Flow cytometry analysis of the Sf9-GagGFP cell population also revealed its substantial heterogeneity in terms of GFP intensity (Fig. 3a). Although a significant number of cells expressed Gag-GFP at relatively high levels, the majority of them had fluorescent intensities similar to parental cells. Therefore, to enrich the population in cells with high expression, we applied a recently developed FACS protocol for shear-sensitive insect cells (Vidigal et al. 2013). After a round of FACS, the mean fluorescence intensity of the population could be enhanced by almost tenfold (Fig. 3a). Unprocessed and matured Gag could be detected by Western blot in culture supernatants of sorted cells, and both seem to be significantly higher than in the non-sorted population (Fig. 3b). This was confirmed by ELISA where a sixfold increase in p24 protein could be observed (Fig. 3c).

The traditional step after selection of a stable polyclonal cell population consists in single-cell cloning and screening for high producers, a labor-intensive and time-consuming process that can take more than 8 weeks (Zitzmann et al. 2017). In this study, rather than starting screening for clones with singlecopy integration of the tagging cassette, the Sf9-GagGFP cell population was first submitted to RMCE (Fig. 1a). The aim was to reduce clone screening time by increasing the probability of selecting cells tagged in loci supporting site-specific recombination. The Sf9-GagGFP population was transfected with a cassette encoding a red reporter protein (iCherry) and a Flp recombinase (iFlp), whose genes were codon-optimized for Sf9 cells (Fig. 1b). Flow cytometry analysis of the population 4 days post transfection (dpt) indicated that cassette exchange was well-succeeded, with about $6 \%$ of the cells expressing iCherry (Fig. 4a). This recombination efficiency is within the reported values for a Flp-RMCE process in mammalian cells (Qiao et al. 2009), suggesting that the FRT site within the linker between the two genes does not have impact on recombination. Due to the two-promoter traps, only cells that exchanged cassettes will express iCherry and resist to G418 selection. After 3 weeks in antibiotic selection, flow 
A)

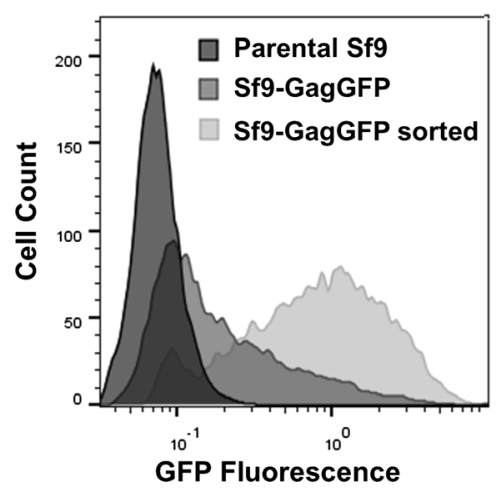

Fig. 3 Isolation of high GagGFP-producing Sf9 cells by FACS. a Flow cytometry analysis of the Sf9-GagGFP cell pools, before and after sorting for high GagGFP-expressing cells. Parental Sf9 cells were used as negative control. b Western blot analysis and c ELISA of p24 Gag

cytometry data shows that the percentage of iCherryexpressing cells could be enhanced to $60 \%$ (Fig. 4a), and fluorescence microscopy of the monolayer cultures show red as well as green colonies (Fig. 4b). To get rid of resistant GFPexpressing cells, the $\mathrm{Sf} 9-\mathrm{Gag} C h e r r y$ population was harvested and sorted for GFP-negative cells.

\section{Establishment of the RMCE Sf9-Gag cell line}

\section{Screening of Sf9-GagCherry cell clones}

We next isolated single cells from the Sf9-GagCherry population by sorting for GFP-negative and iCherry-positive cells, which were collected into 96-well plates. The expansion of the colonies was monitored regularly; all wells containing multiple colonies were discarded. Cell growth performance and iCherry fluorescence intensity were characterized for seven of the isolated clones. Higher growth rates and peak densities were observed for cell clones \#10, \#11, \#12, and \#13 (Fig. 5a). Noteworthy, these four clones had also improved iCherry fluorescence distributions (Fig. 5b). Therefore, they were
C)

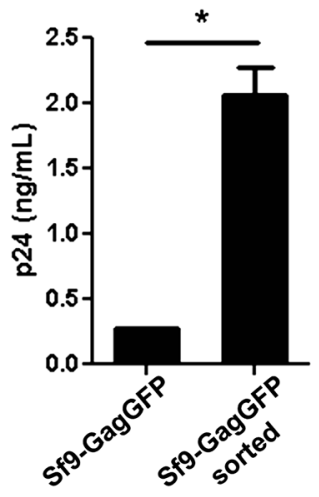

accumulated in the culture supernatant (192 h post inoculation) of populations before and after sorting. ELISA statistical significance was determined on the three independent experiments using the two-tailed Student $t$ test, $* p<0.01$

selected for further characterization in terms of cassette copy number by real-time PCR and Gag expression levels by ELISA. Real-time PCR data confirmed that all four clones have a single targetable locus (Fig. 5c), a desired feature to achieve a more robust RMCE cell line. Concerning productivity assessment, clone \#11 showed significantly higher Gag expression levels than the remaining three clones (Fig. 5d). We also performed genomic PCR analysis of clone \#11 to confirm that the target cassette had been introduced by RMCE (Fig. S2). Based on this data, we selected clone \#11 for a proof-of-concept study, in which we explored the developed RMCE cell platform (Sf9-GagCherry-11 cell line) to produce Gag-VLPs decorated with a membrane protein.

\section{Co-expression of Gag and $\beta 2 A R$ proteins from the same locus}

As target membrane protein, $\beta 2 \mathrm{AR}$ fused to a reporter protein (citrine) and to a hemagglutinin tag (HA-tag) was used to facilitate the detection of both (i) cells co-expressing $\beta 2 \mathrm{AR}$ and Gag proteins upon Flp recombination and (ii) $\beta 2 A R$ on the surface of Gag-VLPs secreted into the culture supernatant.

A)

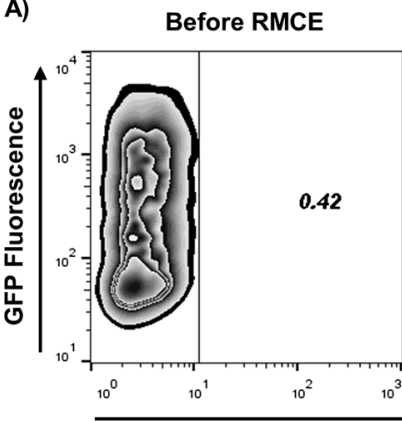

After RMCE (4 dpt)

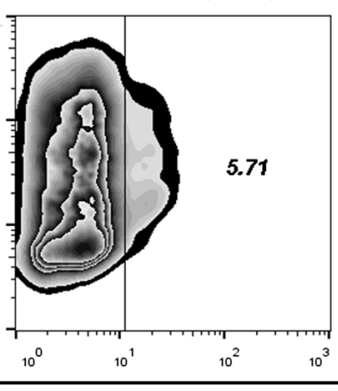

iCherry Fluorescence

Fig. 4 Flipase-mediated cassette exchange to isolate $\mathrm{Sf} 9$ cell clones tagged in targetable loci. a GFP and iCherry flow cytometric profiles of the population before transfection, $96 \mathrm{~h}$ post transfection with the
After RMCE (21 dpt)

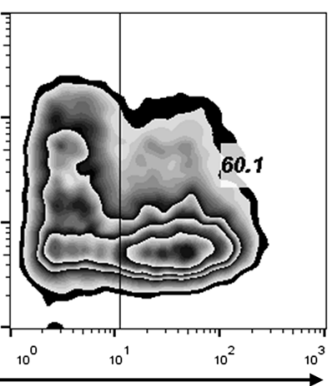

B)

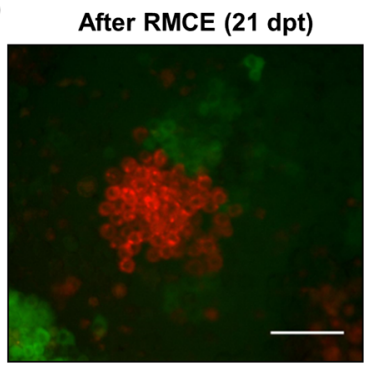

pTarget-Cherry cassette and 3 weeks in G418 selection. b Fluorescence microscopy of the population after Flp-mediated cassette exchange and G418 selection during 3 weeks. Scale bar represents $100 \mu \mathrm{m}$ 
Fig. 5 Characterization of Sf9GagCherry cell clones isolated by FACS. a Growth profiles and $\mathbf{b}$ iCherry fluorescence distribution of cell clones $8,10,11,12,13,14$, and 17. c Copy number assessment by real-time quantitative PCR. d p24 Gag concentration in supernatant samples at the time of harvest assessed by ELISA. Statistical significance from ELISA results was determined based on three independent experiments using the two-tailed Student $t$ test, $* p<0.01$
A)

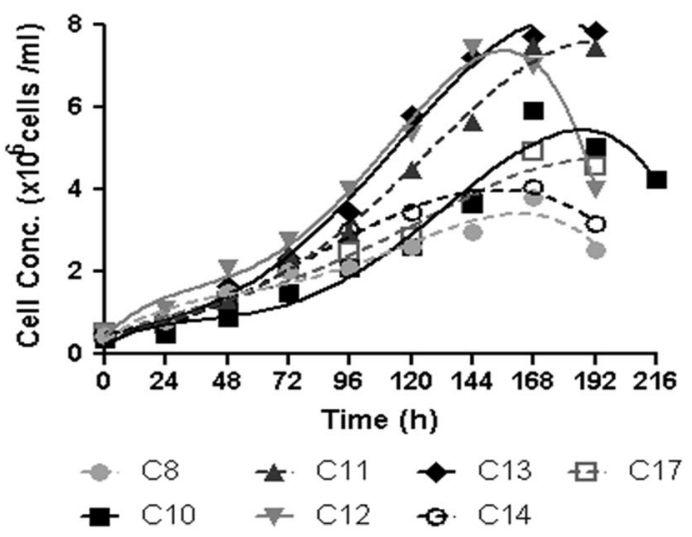

C)

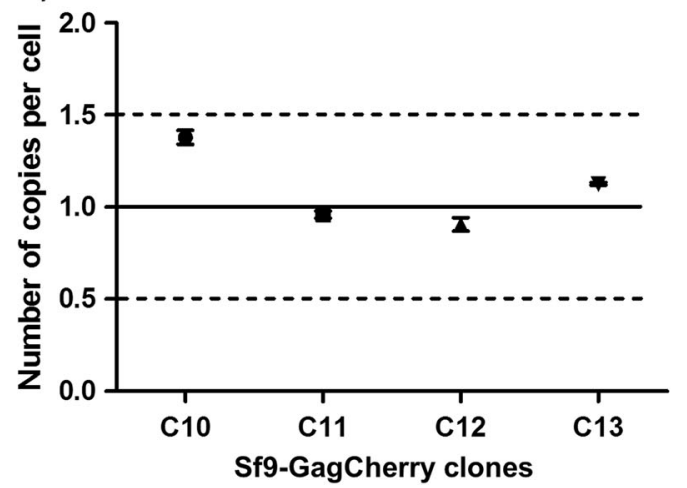

B)

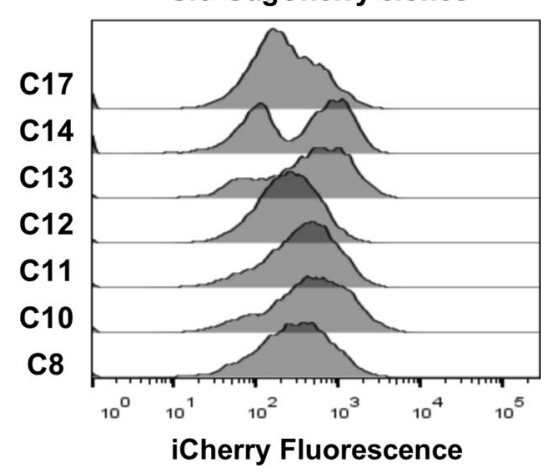

D)

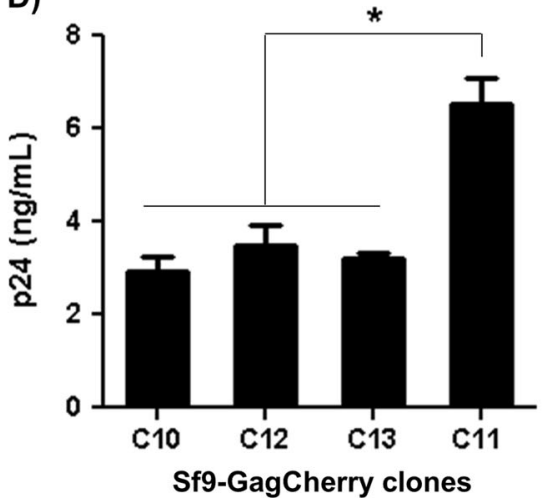

We transfected Sf9-GagCherry-11 cells with the $\beta 2 A R-$ encoding target cassette (Fig. 1b), and eliminated the residual iCherry-expressing cells by FACS after a period of 3 weeks under zeocin (Zeo) selection. An homogeneous population of citrine-positive and iCherry-negative cells was obtained at the end of this process (Fig. 6a). Fluorescence microscopy confirmed the localization of $\beta 2 \mathrm{AR}$-citrine fusion proteins at the plasma membrane (Fig. 6b). Sf9-Gag- $\beta 2$ AR cell growth kinetics closely resemble that of cells from which they were derived (Sf9-GagCherry-11) (Fig. 6c), but the production levels of Gag in Sf9-Gag- $\beta 2$ AR cells as estimated by ELISA were almost threefold higher (Fig. 6d). The integral of viable cells is similar for both cell populations $(0.56$ and 0.49 million cells $\mathrm{mL}^{-1} \mathrm{~h}^{-1}$ ), thus the Gag per cell productivity is also higher in Sf9-Gag- $\beta 2 \mathrm{AR}$ cells. This difference may be directly related with the lower burden imposed to cells by the expression of Gag (in Sf9-Gag- $\beta 2 \mathrm{AR}$ cells) instead of its fusion with iCherry (in Sf9-GagCherry-11 cells), as observed in other expression systems (Larson et al. 2005).

The identification of $\beta 2 \mathrm{AR}$ and Gag proteins was performed by Western blot in cell culture samples concentrated by ultracentrifugation (Fig. 6e). The band corresponding to $\beta 2 \mathrm{AR}$ protein (approx. $110 \mathrm{kDa}$ ) could be detected in Sf9Gag- $\beta 2$ AR cells using an anti-HA antibody. Immunoblotting of Gag from Sf9-Gag- $\beta 2$ AR culture samples detected the cleavage products of the Gag polyprotein, namely, the CA domain $(24 \mathrm{kDa})$, the MA and CA domains $(40 \mathrm{kDa})$, and p55 $(55 \mathrm{kDa})$. The $83-\mathrm{kDa}$ band observed in Sf9GagCherry-11 cells corresponds to the Gag-iCherry fusion protein, which is absent in Sf9-Gag- $\beta 2 \mathrm{AR}$ cells. A nonquantitative ELISA was performed to differentiate between Gag-VLPs (naked VLPs) and 32 AR-displaying Gag-VLPs (full VLPs) measuring the absorbance at $450 \mathrm{~nm}$ (see "Materials and methods" section for details). The full VLPs have threefold higher optical density than naked VLPs, suggesting that $\beta 2 A R$ proteins are being displayed on the surface of the Gag-VLPs (Fig. 6f). We also performed confocal microscopy of concentrated supernatant samples from Sf9GagCherry-11 cells transfected with a $\beta 2 A R$-citrineexpressing plasmid, and could observe the co-localization of Gag and $\beta 2 A R$ on secreted particles (Fig. S3).

\section{Discussion}

Enveloped VLPs have emerged as an efficient and costeffective tool for displaying membrane proteins in their native conformation, to be used as safe and potent vaccines, or on screening platforms for small drug or antibody discovery (Hoffman et al. 2000; Willis et al. 2008). In this work, we 
A)

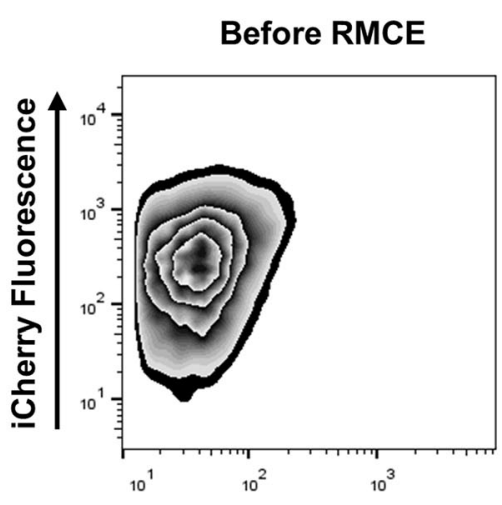

After RMCE (4 dpt)

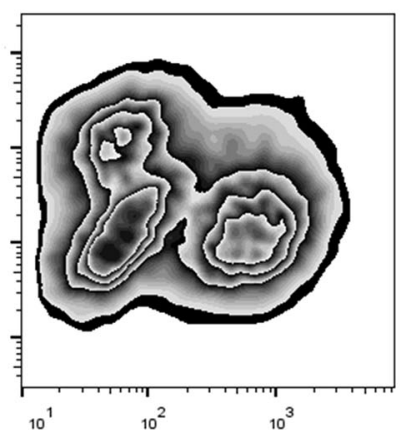

Citrine Fluorescence
After FACS (4 weeks)

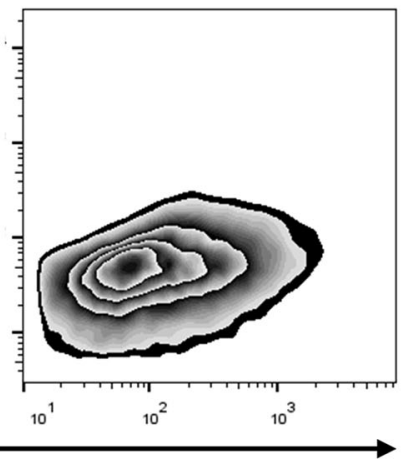

C)
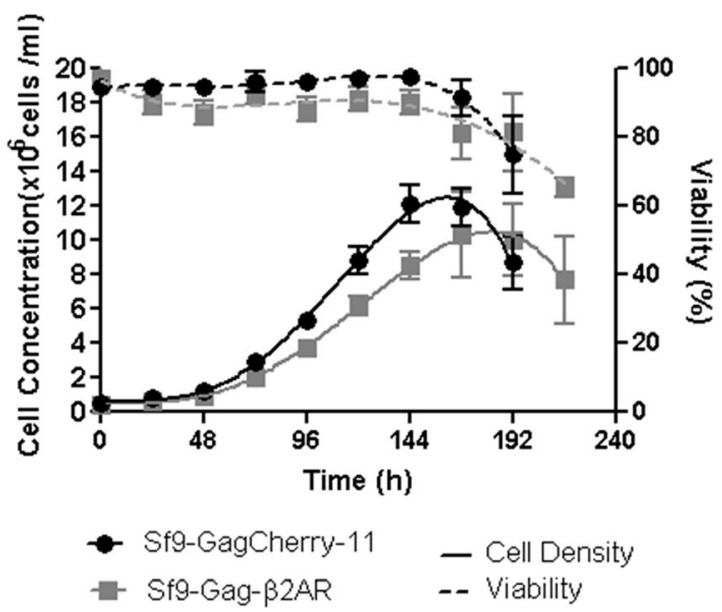

- Sf9-Gag-B2AR _- Viability

F)
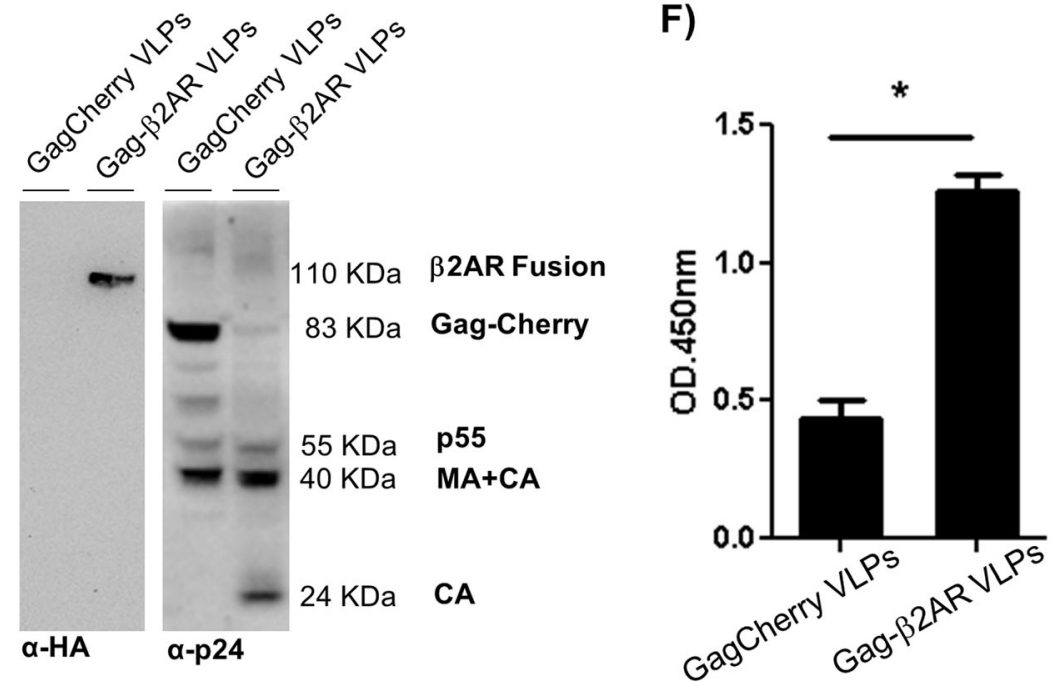

Fig. 6 Flp-mediated cassette exchange to produce $\beta 2$ AR-displaying GagVLPs. a Flow cytometry analysis of clone 11 before transfection, 4 days post transfection (dpt) with pTarget- $\beta 2 \mathrm{AR}$ cassette and 4 weeks under antibiotic selection and FACS to sort out the residual iCherry-positive cells. b Fluorescence microscopy of clone 11 before and after cassette exchange, in which the iCherry fused to Gag was eliminated and cells started to express a citrine fluorescently labeled $\beta 2 \mathrm{AR}$. White arrows point citrine fluorescence mainly at the plasma membrane. The scale bar represents $100 \mu \mathrm{m}$. c Growth profiles and d p24 Gag concentration in supernatant samples at the time of harvest of Sf9-GagCherry-11 and Sf9-Gag- $32 \mathrm{AR}$ cell cultures. Data from three independent experiments. e, f Characterization of Gag-VLPs in ultracentrifuged supernatant samples from Sf9-GagCherry11 and Sf9-Gag- $\beta 2$ AR cell cultures. e Western blot analysis of $\beta 2 A R$ (using a mAb specific to the HA tag in the $\beta 2 \mathrm{AR}$ ) and p24 Gag proteins. Representative pictures from three experiments are shown. f ELISA data from "naked" and $\beta 2$ AR-pseudotyped Gag-VLPs using an anti-HA mAb. The differential absorbance at $450 \mathrm{~nm}$ suggests the incorporation of HAlabeled $\beta 2 A R$ in the Gag-VLPs of the Sf9-Gag- $\beta 2 A R$ cells. Statistical significance was determined based on three independent experiments using the two-tailed Student $t$ test, $* p<0.01$ 
established an insect Sf9 cell line using a Flp-mediated sitespecific recombination system, aimed at accelerating the production of membrane proteins anchored on HIV-1 Gag-VLPs.

RMCE systems have been widely adopted to circumvent the laborious cell line development process associated to random genomic integration of the transgene, which requires extensive clone screening to identify the best performers for each new recombinant protein to be produced. In RMCE systems, a fluorescent reporter protein is typically used in the tagging cassette to facilitate the identification of cell clones tagged in loci supporting better expression levels. However, as fluorescent proteins are synthesized and stay in the cytoplasm, we might end up selecting clones with high transcription rates of the GOI but with an inefficient secretory machinery, which is a recognized bottleneck of cell lines engineered to produce secreted proteins (Peng and Fussenegger 2009; Tigges and Fussenegger 2006). One of the main design principles of our RMCE strategy was to use Gag fused to reporter proteins, to allow highthroughput screening of cells that not only have high Gag expression (due to the 1:1 stoichiometry with the reporter protein), but also provide functional synthesis and maturation of Gag proteins destined to the plasma membrane. As Gag is the common scaffold to expose different membrane proteins of interest, we placed it outside the Flp recombination region, while its fused fluorescent protein was placed inside to be eliminated from the particle by Flp recombination once the cell line had been established. The fusion linker between the two proteins was inserted on the Gag C-terminal domain, based on previous Gag structural and functional studies (Lingappa et al. 2014; Sundquist and Kräusslich 2012), and consisted of one FRT site flanked by two glycine-rich domains. We showed that this unconventional linker does not impair either (i) the formation of Gag-GFP protein complexes, which we could detect at the plasma membrane by fluorescence microscopy and in the culture supernatant by Western blot or (ii) the assembly of Gag proteins as VLPs, which were observed in the culture supernatant by TEM. The fusion of Gag to fluorescent and nonfluorescent proteins has been performed in different ways, including inserting GFP or Cre recombinase between the MA and CA domains of Gag, demonstrating the plasticity and flexibility of the retrovirus core protein as nanocarrier (Dale et al. 2011; Gutiérrez-Granados et al. 2013; Kaczmarczyk et al. 2011; Kawabe et al. 2016).

Achieving genomic integration in high expressing loci after transfection is pivotal for the success of RMCE systems (Baer and Bode 2001; Qiao et al. 2009). Antibiotic selection provides proliferative advantage to those cells that underwent integration, but may also select for expression of its own transcription unit ultimately leading to poor expression of the transgene, even when the expression cassette is placed in close proximity to the antibiotic marker (Kaufman et al. 2008). When populations are isolated by FACS, higher levels of transgene expression can be achieved, as we specifically collect cells with transgenes integrated in a favorable chromatin environment (Kaufman et al. 2008). In this study, we adopted a combination of these two complementary selection procedures, which has proven to improve significantly the cell line development process (Qiao et al. 2009).

Besides providing the best production levels of functional Gag-VLPs, the cell clones should also be Flp-RMCE competent. We have previously established a Flp-mediated cassette exchange system in insect cells in which the majority of the isolated clones were tagged in loci not amenable for recombination, therefore making the cell line development process highly inefficient (Fernandes et al. 2012). Since very little is known about the correlation between high expression levels and RMCE competence, the selection of a clone combining both features is extremely difficult. To get rid of cells tagged in RMCE incompetent loci before single-cell cloning, we added an intermediate step of Flp-mediated cassette exchange in the Sf9 tagging population. By means of two promoter traps, we could use antibiotic resistance and FACS to select cells which are tagged at least in one locus supporting Flp recombination. Through this step, we could also easily confirm that the FRT which integrates the fusion linker remains accessible to the Flp as cells which were GFP positive turned into iCherry positive after Flp recombination. This step of enrichment with cells supporting RMCE has also been adopted in cell engineering studies of Chinese hamster ovary (CHO) cells (Qiao et al. 2009). The co-selection of high Gag-expressing and RMCE competent clones reduced significantly the number of clones we needed to screen to generate a master RMCE cell line.

As proof of concept, we used the best Gag producer clone to co-express a human $\beta 2 \mathrm{AR}$ from its tagged locus, and could confirm the presence of the target protein on the surface of secreted Gag-VLPs by confocal fluorescence microscopy and by ELISA, in which a $\beta 2 A R$-specific antibody bound to the B2AR-displaying VLPs but not to "naked" VLPs (i.e., that do not display the target protein). Capturing such complex receptors in a native conformation, in the membrane-like environment of Gag-VLPs, can be particularly advantageous for antibody selection/generation by phage display or immunization-screening tools, in which antibodies that recognize the VLP scaffold can be easily removed by first providing "naked" VLPs to the phage library/animals before providing the "full" VLPs.

Overall, this work demonstrates the suitability of the RMCE approach adopted to rapidly develop a stable Sf9 cell platform to efficiently produce Gag-VLPs pseudotyped with membrane proteins of interest. It constitutes a valuable expression tool to overcome specific downsides of stable (e.g., random integration) and transient (e.g., insect cells-baculovirus) expression systems, and a fast strategy to produce receptors and viral antigens in a soluble format, to fuel therapeutic antibody/ drug discovery and vaccine development programs. 
Acknowledgements We gratefully thank Dr. Joop van den Heuvel from PSPF at the Helmholtz Centre for Infection Research (Braunschweig, Germany) for providing the OpIE2-iFlp-IEterm plasmid. We also acknowledge the technical support from C. Bispo, C. Andrade, and R. Gardner (Flow Cytometry Facility, Instituto Gulbenkian de Ciência (IGC)) and A.C Correia and E.M. Tranfield (Electron Microscopy Facility, IGC).

Funding information This work was supported by the European Commission FP7 through the "ComplexINC" project (grant agreement no. 270089), and by the Portuguese "Fundação para a Ciência e a Tecnologia" (FCT) through the following initiatives: R\&D Projects (PTDC/EBB-EBI/102266/2008, EXPL/BBB-BIO/1541/2013, and IF/ 01704/2014/CP1229/CT0001), PhD fellowships (SFRH/BD/90564/ 2012 and SFRH/BD/86744/2012), and "Investigador FCT" Program (IF/01704/2014).

Compliance with ethical standards This article does not contain any studies with human participants or animals performed by any of the authors.

Conflict of interest The authors declare that they have no conflict of interest.

\section{References}

Baer A, Bode J (2001) Coping with kinetic and thermodynamic barriers : RMCE, an efficient strategy for the targeted integration of transgenes. Curr Opin Biotechnol 12:473-480. https://doi.org/10. 1016/S0958-1669(00)00248-2

Briggs JAG, Grünewald K, Glass B, Förster F, Kräusslich H-G, Fuller SD (2006) The mechanism of HIV-1 core assembly: insights from threedimensional reconstructions of authentic virions. Structure 14(1): 15-20. https://doi.org/10.1016/j.str.2005.09.010

Chua AJ, Vituret C, Tan ML, Gonzalez G, Boulanger P, Ng M-L, Hong SS (2013) A novel platform for virus-like particle-display of flaviviral envelope domain III: induction of dengue and West Nile virus neutralizing antibodies. Virol J 10(1):129. https://doi.org/10.1186/1743422X-10-129

Cruz PE, Peixoto CC, Devos K, Moreira JL, Saman E, Carrondo MJT (2000) Characterization and downstream processing of HIV-1 core and virus-like particles produced in serum free medium. Enzym Microb Technol 26(1):61-70. https://doi.org/10.1016/S01410229(99)00128-3

Dale BM, McNerney GP, Hübner W, Huser TR, Chen BK (2011) Tracking and quantitation of fluorescent HIV during cell-to-cell transmission. Methods 53(1):20-26. https://doi.org/10.1016/j. ymeth.2010.06.018

Delchambre M, Gheysen D, Thines D, Thiriart C, Jacobs E, Verdin E, Horth M, Burny A, Bex F (1989) The GAG precursor of simian immunodeficiency virus assembles into virus-like particles. EMBO J 8(9):2653-2660. https://doi.org/10.1002/j.1460-2075.1989. tb08405.x

Fernandes F, Teixeira AP, Carinhas N, Carrondo MJ, Alves PM (2013) Insect cells as a production platform of complex virus-like particles. Expert Rev Vaccines 12(2):225-236. https://doi.org/10.1586/erv.12.153

Fernandes F, Vidigal J, Dias MM, Prather KLJ, Coroadinha AS, Teixeira AP, Alves PM (2012) Flipase-mediated cassette exchange in Sf9 insect cells for stable gene expression. Biotechnol Bioeng 109(11): 2836-2844. https://doi.org/10.1002/bit.24542

Gutiérrez-Granados S, Cervera L, Gòdia F, Carrillo J, Segura MM (2013) Development and validation of a quantitation assay for fluorescently tagged HIV-1 virus-like particles. J Virol Methods 193(1):85-95. https://doi.org/10.1016/j.jviromet.2013.05.010

Harrison RL, Jarvis DL (2016) Transforming lepidopteran insect cells for continuous recombinant protein expression. Baculovirus and Insect Cell Expression Protocols 329-348. https://doi.org/10.1007/978-14939-3043-2_16

Haynes JR, Dokken L, Wiley JA, Cawthon AG, Bigger J, Harmsen AG, Richardson C (2009) Influenza-pseudotyped Gag virus-like particle vaccines provide broad protection against highly pathogenic avian influenza challenge. Vaccine 27(4):530-541. https://doi.org/10. 1016/j.vaccine.2008.11.011

Hoffman TL, Canziani G, Jia L, Rucker J, Doms RW (2000) A biosensor assay for studying ligand-membrane receptor interactions: binding of antibodies and HIV-1 Env to chemokine receptors. Proc Natl Acad Sci U S A 97(21):11215-11220. https://doi.org/10.1073/ pnas. 190274097

Huang R, Kiss M, Batonick M, Weiner M, Kay B (2016) Generating recombinant antibodies to membrane proteins through phage display. Antibodies 5(2):11. https://doi.org/10.3390/antib5020011

Hutchings CJ, Koglin M, Olson WC, Marshall FH (2017) Opportunities for therapeutic antibodies directed at G-protein-coupled receptors. Nat Rev Drug Discov In press 16(11):787-810. https://doi.org/10. 1038/nrd.2017.91

Hutchings CJ, Koglin M, Marshall FH (2010) Therapeutic antibodies directed at $\mathrm{G}$ protein-coupled receptors. MAbs 2(6):594-606. https://doi.org/10.4161/mabs.2.6.13420

Kaczmarczyk SJ, Sitaraman K, Young HA, Hughes SH, Chatterjee DK (2011) Protein delivery using engineered virus-like particles. Proc Natl Acad Sci U S A 108(41):16998-17003. https://doi.org/10. 1073/pnas.1101874108

Kaufman WL, Kocman I, Agrawal V, Rahn H-P, Besser D, Gossen M (2008) Homogeneity and persistence of transgene expression by omitting antibiotic selection in cell line isolation. Nucleic Acids Res 36(17):e111. https://doi.org/10.1093/nar/gkn508

Kawabe Y, Shimomura T, Huang S, Imanishi S, Ito A, Kamihira M (2016) Targeted transgene insertion into the $\mathrm{CHO}$ cell genome using Cre recombinase-incorporating integrase-defective retroviral vectors. Biotechnol Bioeng 113(7):1600-1610. https://doi.org/10. 1002/bit.25923

Kirchmeier M, Fluckiger AC, Soare C, Bozic J, Ontsouka B, Ahmed T, Diress A, Pereira L, Schodel F, Plotkin S, Dalba C, Klatzmann D, Anderson DE (2014) Enveloped virus-like particle expression of human cytomegalovirus glycoprotein B antigen induces antibodies with potent and broad neutralizing activity. Clin Vaccine Immunol 21(2):174-180. https://doi.org/10.1128/CVI.00662-13

Kurg R, Reinsalu O, Jagur S, Õunap K, Võsa L, Kasvandik S, Padari K, Gildemann K, Ustav M (2016) Biochemical and proteomic characterization of retrovirus Gag based microparticles carrying melanoma antigens. Sci Rep 6:29425. https://doi.org/10.1038/srep29425

Larson DR, Johnson MC, Webb WW, Vogt VM (2005) Visualization of retrovirus budding with correlated light and electron microscopy. Proc Natl Acad Sci U S A 102(43):15453-15458. https://doi.org/ 10.1073/pnas.0504812102

Lingappa JR, Reed JC, Tanaka M, Chutiraka K, Robinson BA (2014) How HIV-1 Gag assembles in cells: putting together pieces of the puzzle. Virus Res 193:89-107. https://doi.org/10.1016/j.virusres. 2014.07.001

Patrone M, Coroadinha AS, Teixeira AP, Alves PM (2016) Palmitoylation strengthens cholesterol-dependent multimerization and fusion activity of human cytomegalovirus glycoprotein B (gB). J Biol Chem 291(9): 4711-4722. https://doi.org/10.1074/jbc.M115.682252

Peng RW, Fussenegger M (2009) Molecular engineering of exocytic vesicle traffic enhances the productivity of Chinese hamster ovary cells. Biotechnol Bioeng 102(4):1170-1181. https://doi.org/10.1002/bit. 22141 
Pushko P, Tretyakova I, Hidajat R, Zsak A, Chrzastek K, Tumpey TM, Kapczynski DR (2017) Virus-like particles displaying H5, H7, H9 hemagglutinins and $\mathrm{N} 1$ neuraminidase elicit protective immunity to heterologous avian influenza viruses in chickens. Virology 501: 176-182. https://doi.org/10.1016/j.virol.2016.12.001

Qiao J, Oumard A, Wegloehner W, Bode J (2009) Novel tag-andexchange (RMCE) strategies generate master cell clones with predictable and stable transgene expression properties. J Mol Biol 390(4):579-594. https://doi.org/10.1016/j.jmb.2009.05.012

Sundquist WI, Kräusslich H-G (2012) HIV-1 assembly, budding, and maturation. Cold Spring Harb Perspect Med 2(7):a006924. https:// doi.org/10.1101/cshperspect.a006924

Takeda H, Ogasawara T, Ozawa T, Muraguchi A, Jih P-J, Morishita R, Uchigashima M, Watanabe M, Fujimoto T, Iwasaki T, Endo Y, Sawasaki T (2015) Production of monoclonal antibodies against GPCR using cell-free synthesized GPCR antigen and biotinylated liposome-based interaction assay. Sci Rep 5(1):11333. https://doi. org $/ 10.1038 /$ srep 11333

Tigges M, Fussenegger M (2006) Xbp1-based engineering of secretory capacity enhances the productivity of Chinese hamster ovary cells. Metab Eng 8(3):264-272. https://doi.org/10.1016/j.ymben.2006.01.006

Turan S, Qiao J, Madden S, Benham C, Kotz M, Schambach A, Bode J (2014) Expanding Flp-RMCE options: the potential of Recombinase Mediated Twin-Site Targeting (RMTT). Gene 546(2):135-144. https://doi.org/10.1016/j.gene.2014.06.002
Turan S, Zehe C, Kuehle J, Qiao J, Bode J (2013) Recombinase-mediated cassette exchange (RMCE) - a rapidly-expanding toolbox for targeted genomic modifications. Gene 515(1):1-27. https://doi.org/ 10.1016/j.gene.2012.11.016

Ulmer JB, Valley U, Rappuoli R (2006) Vaccine manufacturing: challenges and solutions. Nat Biotechnol 24(11):1377-1383. https:// doi.org/10.1038/nbt1261

van Oers MM (2011) Opportunities and challenges for the baculovirus expression system. J Invertebr Pathol 107(Suppl):S3-S15. https:// doi.org/10.1016/j.jip.2011.05.001

Vidigal J, Dias MM, Fernandes F, Patrone M, Bispo C, Andrade C, Gardner R, Carrondo MJT, Alves PM, Teixeira AP (2013) A cell sorting protocol for selecting high-producing sub-populations of Sf9 and High Five ${ }^{\mathrm{TM}}$ cells. J Biotechnol 168(4):436-439. https://doi. org/10.1016/j.jbiotec.2013.10.020

Willis S, Davidoff C, Schilling J, Wanless A, Doranz BJ, Rucker J (2008) Virus-like particles as quantitative probes of membrane protein interactions. Biochemistry 47(27):6988-6990. https://doi.org/10. 1021/bi800540b

Zhao Q, Wu B (2012) Ice breaking in GPCR structural biology. Acta Pharmacol Sin 33(3):324-334. https://doi.org/10.1038/aps.2011.187

Zitzmann J, Sprick G, Weidner T, Schreiber C, Czermak P (2017) Process optimization for recombinant protein expression in insect cells, new insights into cell cult. Technol., Dr. Sivakumar Joghi Thatha Gowder (Ed.), InTech, https://doi.org/10.5772/67849 